\title{
Impact Evaluation of Comfort Care on the Prognosis of Giant Thoracic Tumor Patients with Computerized Tomography Imaging under Intelligent Reconstruction Algorithm
}

\author{
Yan Li (iD \\ Cardiothoracic Surgery Ward 2, The Third Affiliated Hospital of Qiqihar Medical University, Qiqihar 161000, \\ Heilongjiang, China \\ Correspondence should be addressed to Yan Li; f10012211@st.sandau.edu.cn
}

Received 23 November 2021; Revised 12 January 2022; Accepted 13 January 2022; Published 8 February 2022

Academic Editor: M. Pallikonda Rajasekaran

Copyright (C) 2022 Yan Li. This is an open access article distributed under the Creative Commons Attribution License, which
permits unrestricted use, distribution, and reproduction in any medium, provided the original work is properly cited.

The reconstruction algorithm based on the network of generative adversarial and contextual coding (RANGC) was proposed in this study to analyze the impacts on the prognosis of patients with giant thoracic tumors with CT imaging under artificial intelligence algorithms. The algorithms of Feldkamp-Davis-Kress (FDK) and the generative adversarial network (GAN) were introduced. The patients were divided into the test group with comfort care and the control group with conventional care. Three sets of indicators below were also compared between the patients in two groups, including the pain level and complication incidence, the self-rating anxiety scale (SAS), the self-rating depression scale (SDS), and the patients' satisfaction and average duration of hospital stay. When the scanning range was $\left[0^{\circ}, 89^{\circ}\right]$, the peak signal-to-noise ratio (PSNR) and structural similarity index measurement (SSIM) under the RANGC algorithm were $45.6 \mathrm{~dB}$ and 0.99 , respectively. When the scanning range changed to $\left[0^{\circ}, 119^{\circ}\right]$, the PSNR and SSIM were $39.21 \mathrm{~dB}$ and 0.98 , respectively. The results were significantly higher than those under the FDK and GAN algorithms, and the difference was obviously of statistical significance $(P<0.05)$. The average postoperative pain level of the patients in the control group was 3.12 points, and the postoperative complication incidence was $36.13 \%$, while those of the test group patients were 2.27 points and $20.02 \%$, respectively, which was greatly lower than those of the control group patients, and such a difference was of statistical significance $(P<0.05)$. There was no statistically significant difference in the SDS and SAS scores between the patients in the two groups before surgery. However, the SAS and SDS scores of the test group patients were 41.23 and 43.25, respectively, after surgery, which are obviously lower than those of the control group patients, with a statistically significant difference $(P<0.05)$. The average duration of hospital stay of the test group patients was 6.31 days, which was lower than that of the control group patients, with a statistically significant difference $(P<0.05)$. The overall satisfaction of the test group patients was $83.33 \%$, which was remarkably higher than that of the control group patients, and the difference had statistical significance $(P<0.05)$. All these showed that the performance of the RANGC algorithm was relatively better, and comfort care did good to improve the negative mood, satisfaction, and life quality of patients after surgery.

\section{Introduction}

A giant thoracic tumor is a kind of rare tumor clinically [1-3]. Because of its complicated pathological types and prognosis, there is no unified standard for the diagnosis and treatment, and surgical resection becomes the major clinical treatment means [4-7]. The specific location, origin, and adjacency relations of the tumor can be assessed accurately by an imaging examination. The tumor adhesion and blood supply can be identified, and the feasibility of complete surgical resection would be determined $[8,9]$. Comfort care is a holistic, creative, and personalized nursing model, aiming to make people pleasant at the psychological, physical, and social communication levels. Ye et al. (2021) [10] explored the impact of comfort care on the recovery quality of patients with lung cancer. As a result, comfort care reduced complications during the recovery effectively and was worthy of clinical application. 
Computed tomography (CT) is a commonly applied imaging method at present. However, conventional CT provides only the axial cross-sectional images, which requires the doctors to have a rich clinical diagnosis experience and spatial imagination. Hence, there are some shortcomings to its objectivity and accuracy $[11,12]$. With the development of imaging technology, CT three-dimensional reconstruction technology is gradually deployed in clinical applications. Through the technology, CT scanned images are superimposed together, and a visual three-dimensional image model is synthesized. The model intuitively presents the shape of the tumor and the relation with the surrounding tissues, giving more accurate guidelines for preoperative evaluation and surgical planning [13-15]. However, there is radiation in most imaging examinations. Some studies have proposed that the radiation can be cut down by reducing the rotation angle during CT scanning. Although it is effective, it has the disadvantage of loss of projection data, which lowers the quality of the reconstructed image and often fails to meet the clinical diagnosis criteria [16].

The intelligent algorithms have been improved, developed, and applied in medical imaging. It has been proposed to unitize the Feldkamp-Davis-Kress (FDK) algorithm for CT images reconstruction in clinical practice. However, investigations have shown that the CT images that come out have poor quality and cannot be used for clinical diagnosis when the FDK algorithm works on reconstructing the image of incomplete cone-beam computerized tomography (CBCT) $[17,18]$. For the superiority of generative adversarial network (GAN) in image processing, the reconstruction algorithm based on the network of generative adversarial and contextual coding (RANGC) was raised in this study and was used in CT imaging evaluation of giant thoracic tumors [19].

CT three-dimensional reconstruction technology is of great significance in the surgical treatment of giant thoracic tumors. GAN is also effective in CT image processing. Therefore, the RANGC algorithm was proposed and used with the CT images for the impact evolution of comfort care on the prognosis of giant thoracic tumor patients. The images under the FDK and GAN algorithms were compared to obtain effective data support for the clinical care of giant thoracic tumor patients.

\section{Materials and Methods}

2.1. Research Objects. In this study, 24 patients with a giant thoracic tumor, who underwent surgical treatment in the hospital from June 2019 to June 2020, were chosen. All the patients, 13 males and 11 females, were diagnosed with a giant thoracic tumor. They were between 16 and 76 years old, and their average age was $43.26 \pm 12.45$ years. Meanwhile, the relevant clinical data of them were collected. As the inclusion criteria requested, the symptom of the included patients should meet the diagnostic criteria for giant thoracic tumors (the longest diameter of the tumor was greater than $10 \mathrm{~cm}$, and the tumor volume occupied more than $40 \%$ of the hemithoracic cavity). At the same time, the included patients had not been treated with neoadjuvant radiotherapy or chemotherapy, and they could offer the relevant clinical materials completely. As for the exclusion criteria, the patients in the following situations were excluded: firstly, the patients who suffered from malignant tumors of other systems, and secondly, the patients who were pregnant and the patients who had poor compliance and could not cooperate with the experiment. This study had been approved by the Ethics Committee of the Hospital, and the patients and their families had known the research situation and signed the informed consents.

2.2. Preoperative Tests. All patients underwent CT scanning of the chest, upper abdominal plain scan, and enhanced examination before surgery. CT scanner was used, and the scanning parameters included $130 \mathrm{KV}, 200 \mathrm{~mA}, 1 \mathrm{r} / \mathrm{s}$, slice thickness $5 \mathrm{~mm}$, interval $1.5 \mathrm{~mm}$, and pitch 1.3. CT-guided percutaneous puncture biopsy was adopted to determine the type of the tumor. Blood gas analysis, blood routine, liver and kidney functions, coagulation function, routine pretransfusion, and other preoperative tests were also performed. CT three-dimensional reconstruction was to know the origin, direction, and relationship with the surrounding tissues of the main nourishing vessels of the tumor to design surgical incisions and intraoperative planning.

2.3. Comfort Care. The 24 patients were randomly divided into two groups. The test group received comfort care, and the control group received conventional care. There was no statistically significant difference in age, gender, and education level among the patients in the two groups $(P>0.05)$. Comfortable environment, comfortable postures, comfortable psychological condition, pain care, diet care, and drainage tube care were included in the comfort care. For a comfortable environment, the wards were kept quiet, clean, and tidy. Professionals were arranged for regular disinfection. The wards were well-ventilated regularly for keeping the air fresh. The temperature, humidity, and light in the wards were all suitable. Nurses helped the patients to have an appropriate posture according to the specific postoperative situation, assisted the patients to change posture, and massaged the lower limbs regularly. With the friendly appearance and attitude, much more communication was made with the patients themselves and their families. When the patients experienced anxiety, depression, and other bad emotions, they were inspired in time. Some successful cases and related pieces of knowledge were introduced to the patients and their families to give them confidence and quell their fear of disease. The pain was an important factor leading to the declining postoperative comfort. The corresponding measures to distract the patients' attention were taken, such as playing music that the patients liked and talking about what the patients were interested in. The patients in the recovery stage needed intensive nourishment, and they were told to eat more fruits, vegetables, and highprotein foods based on their conditions. All the drainage tubes, drainage bags, infusion tubes, and so on were placed properly to keep the drainage tubes unobstructed and to keep them from twisting, discounting, and falling off. 
2.4. Reconstruction Algorithm under Generative Adversarial Network and Contextual Coding Network. It was given that the patients' lives were threatened by the radiation of conventional CT scanning, and low-dose CT has become one of the important trends in CBCT. The low-dose CT reconstruction algorithms are mainly classified into two categories. The first is to aim at the images directly, while the second is to directly aim at the projection data. The theoretical basis of the RANGC algorithm in this study was derived from the second one.

GAN was composed of a generating network $(G n)$ and a discerning network $(D n)$. The $G n$, by learning the probability distribution of the real data in the train set continuously, converted the input random noise into the data that is highly similar to those in the train set. $D n$ was designed for the judgment of whether the data was real, and it made a distinction between the highly similar data in $G n$ and the data in the train set.

The RANGC algorithm was composed of three phases, including data preprocessing, data training, and data testing sequentially, as shown in Figure 1. In the data preprocessing stage, the $\mathrm{CBCT}$ three-dimensional projection data was transformed into two-dimensional data that can be encoded. Then, in the training phase, the two-dimensional data as the input image was used to train $\mathrm{Gn}$, which predicted the missing parts of the image. The real two-dimensional data and the missing part predicted were trained in Dn, and the true probability of the data generated by $\mathrm{Gn}$ was worked out.

The encoder adopted the AlexNet network structure of the convolutional neural network (CNN).

In the data training phase, the complete imaging data set $D$ was input to RANGC. The incomplete imaging data A was carried out for feature extraction in $\mathrm{Gn}$, and the losing part $A^{\prime}$ of the incomplete imaging data was the output. $D$, which is the real imaging data of the losing part of incomplete imaging data, was input to $D n$, and $D n(d)$ was the output, which indicated that the data $d$ was the real imaging data. The training phase was just a process of adversarial learning via Minmax between Dn and Gn, and the adversarial objective function of this process was defined as $K(D n, G n)$. The equation was expressed as follows:

$$
\min _{G n} \max _{D n} K(D n \cdot G n)=E_{d \in D}\left[\log D n(d)+\log \left(1-D n A^{\prime}\right)\right] .
$$

Since it was difficult for the adversarial objective function to take the local details of the generated data into account, the reconstruction objective function was used in this study to make the data predicted by the contextual encoder more reliable. The reconstruction objective function was defined as $P(d)$, and its equation was expressed as follows:

$$
P(d)=\|d-A\|_{2} .
$$

In the test phase, the $\mathrm{CT}$ image could be reconstructed from the complete CT three-dimensional imaging data by the commonly used FDK algorithm in clinical practice. As for the detailed process, the CT three-dimensional imaging data multiplied the cosine function $\cos \alpha$, and the ramp filtering was done on the processed data line-by-line. Then, the filtered data was subjected to cone-beam weighted inverse imaging.

2.5. Observation Indicators. In the seven days before and the two weeks after the surgery, the self-rating anxiety scale (SAS) and the self-rating depression scale (SDS) were adopted for the evaluation of the patients' psychological condition. The patient care quality questionnaires were filled out for the patient's satisfaction with care. The total score was 10 points, and $\leq 6$ points suggested the care was unsatisfactory. When the score fell between 6.1 and 7.5 points, the care was classified to be general, 7.6-8.5 points were classified to be satisfied, and 6.7-10 points were classified to be very satisfied. The overall satisfaction equation was as follows:

Total satisfaction $=\frac{\text { Very satisfied }+ \text { Satisfied }}{\text { Total people }} \times 100 \%$.

2.6. Image Evaluation Indicators. The evaluation of image quality was mainly described by the parameters, such as peak signal-to-noise ratio (PSNR) and structural similarity index measurement (SSIM), which were used to describe each algorithm quantitatively.

PSNR depicted the amount of noise in the image after noise reduction. Therefore, the greater the PSNR value, the lesser the image noise and the better the noise reduction effect.

$$
\mathrm{PSNR}=10 \log _{10} \frac{255^{2}}{\mathrm{MSE}}
$$

Image similarity was mainly applied to compare the similarity of the content between the two images, which was just SSIM with a value range of $[0,1]$. The similarity was judged according to the comparison result. The larger the SSIM value, the better the image quality. If $m$ and $n$ represented the original image and the image to be evaluated, respectively, the average value $(\alpha m, \alpha n)$ indicated the brightness of the image, $(\rho m, \rho n)$ represented the contrast of the image, and $\rho m n$ was the structure of the image. The calculation equation was expressed as follows:

$$
\operatorname{sim}(m, n)=\frac{\left(2 \alpha_{m} \alpha_{n}+G_{1}\right)\left(2 \rho_{m n}+G_{2}\right)}{\left(\alpha_{m}^{2}+\alpha_{n}^{2}+G_{1}\right)\left(\rho_{m}^{2}+\rho_{n}^{2}+G_{2}\right)} .
$$

2.7. Statistical Analysis. SPSS 21.0 was used for data processing and analysis. The measurement data of the serum levels of the three tumor markers were indicated as $( \pm s)$, and the comparison between the groups was performed by $t$-test. The diagnostic efficacy, like sensitivity, specificity, and accuracy, could be taken as count data, and measured as a rate. Chi-square test was done as well for the comparison between the groups, and $P<0.05$ denoted that the comparative difference was statistically significant. 


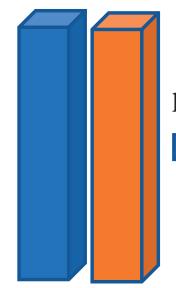

A B

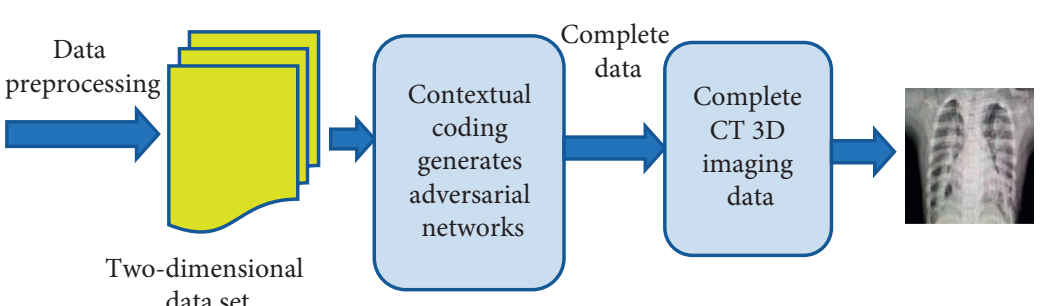

(a)

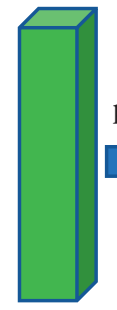

C

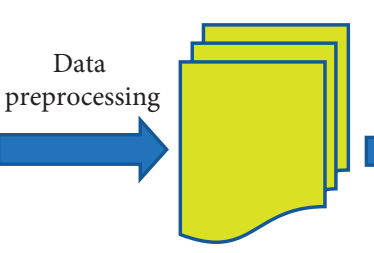

Two-dimensional data set

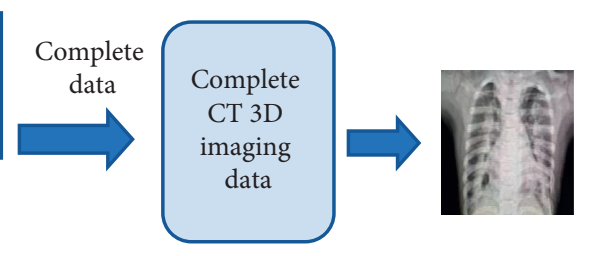

(b)

Figure 1: The algorithm flow of RANGC. (A was the low-dose CT three-dimensional imaging data; B was the real CT three-dimensional imaging data; $\mathrm{C}$ was the low-dose CT three-dimensional imaging data in the test set.)

\section{Results}

3.1. Algorithm Performance Analysis. To verify the performance of the algorithm, PSNR and SSIM were compared when the FDK [20] and GAN [21] algorithms were run in the scanning range of $\left[0^{\circ}, 89^{\circ}\right]$ and $\left[0^{\circ}, 119^{\circ}\right]$. The results were shown in Figure 2 and Figure 3. In Figure 2, PSNR and SSIM of the RANGC algorithm were $45.6 \mathrm{~dB}$ and 0.99 , respectively, which were greatly higher than the results under the FDK and GAN algorithms, and the difference was obviously statistically significant $(P<0.05)$.

In Figure 3, PSNR and SSIM of the RANGC algorithm were $39.21 \mathrm{~dB}$ and 0.98 , respectively, which were also distinctly higher than those of the FDK and GAN algorithms. The difference was obviously with a statistical significance $(P<0.05)$. However, compared to the results in the scanning range of $\left[0^{\circ}, 89^{\circ}\right]$, PSNR was reduced by $14.6 \%$, and SSIM was reduced by $2 \%$.

In the scanning range of $\left[0^{\circ}, 89^{\circ}\right]$, the reconstruction results of the three algorithms were compared. As shown in Figure 4, the image under the RANGC algorithm had a higher quality significantly than that under the FDK and GAN algorithm.

3.2. General Clinical Data of the Patients in Two Groups. As shown in Table 1, the general clinical data of the patients in the two groups were compared. There were 12 patients in the test group ( 6 males and 6 females), with an average age of 42.65 years old and an average education year of 6.3 years. It could be viewed in Figure 5 that compared with those of the control group, there was no statistical significance $(P>0.05)$.
3.3. Results of Comfort Care. The pain level, complication incidence, anxiety and depression score scale, patient satisfaction, and duration of hospital stay of the patients in the two groups were compared. The compared results are shown in Figure 5-7.

Figure 5 shows the pain level and complication incidence of the patients. The average postoperative pain level of the patients in the test group was 2.27 points, and the complication incidence was $20.02 \%$. It was significantly lower than that of the control group, and the difference was statistically significant $(P<0.05)$.

Figure 6(a) shows the comparison of the preoperative scores of SAS and SDS of the patients, while figure 6(b) showed the comparison of those after the surgery. There was no statistical significance of the difference between the patients' preoperative scores in two groups $(P>0.05)$. However, the SAS and SDS scores of the test group patients were 41.23 and 43.25 , respectively, which were obviously lower than those of the control group patients, and the difference was statistically significant $(P<0.05)$.

Figure 7 (a) was the comparison of patient satisfaction between the two groups, and Figure $7(\mathrm{~b})$ is the comparison of the duration of hospital stay. As shown in Figure 7(a), among the patients in the test group, 3 people were very satisfied, 7 people were satisfied, and 2 people were generally satisfied. The overall satisfaction reached $83.33 \%$, while the overall satisfaction of the control group was $41.2 \%$, which was significantly lower than that of the test group. There was statistical significance $(P<0.05)$. In Figure $7(b)$, the patients' average duration of hospital stay in the test group was 6.31 days, and that in the control group reached 8.75 days. The difference was evident and statistically significant $(P<0.05)$. 


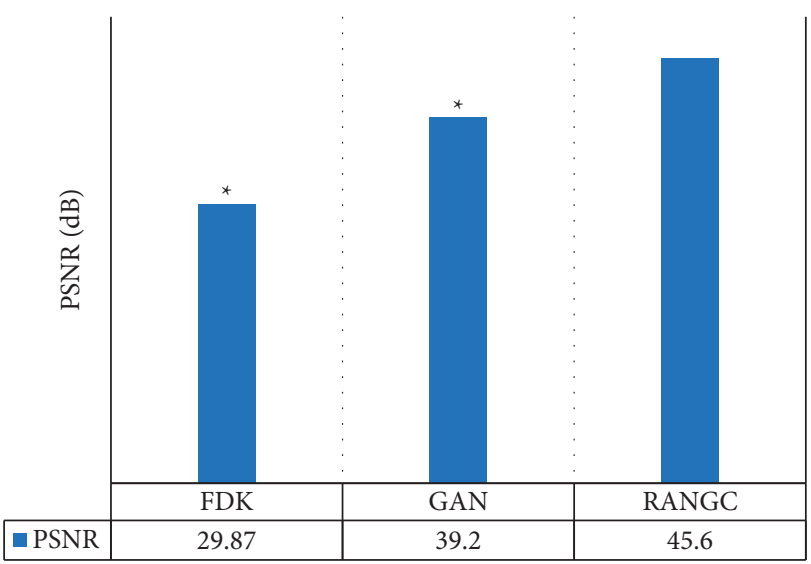

(a)

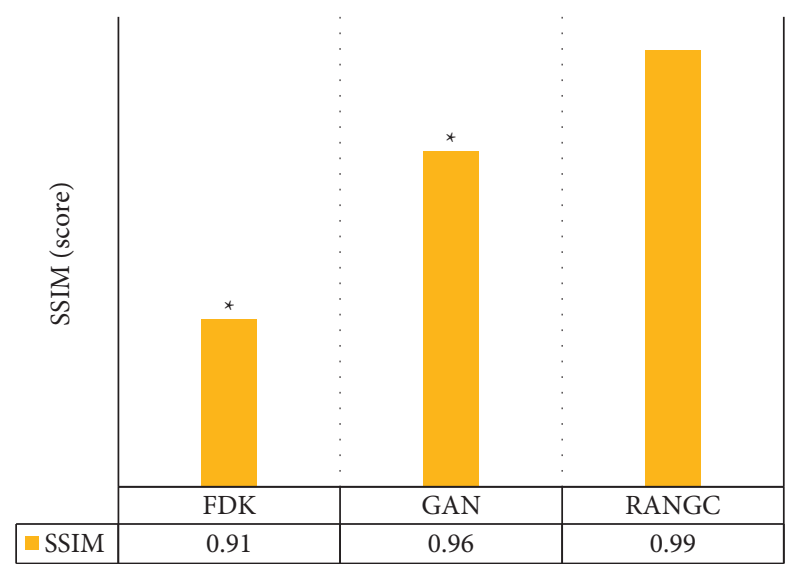

(b)

FIGURE 2: Performance analysis of the three algorithms in the scanning range $\left[0^{\circ}, 89^{\circ}\right]$. Notes: (a) was the results of PSNR and (b) was that of SSIM. ${ }^{*}$ indicated that the difference compared to the result of RANGC was statistically significant $(P<0.05)$.

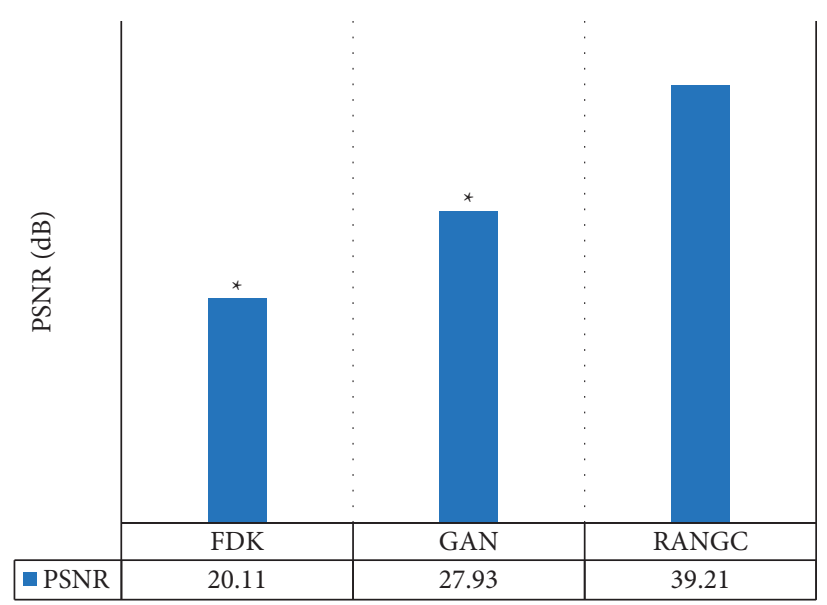

(a)

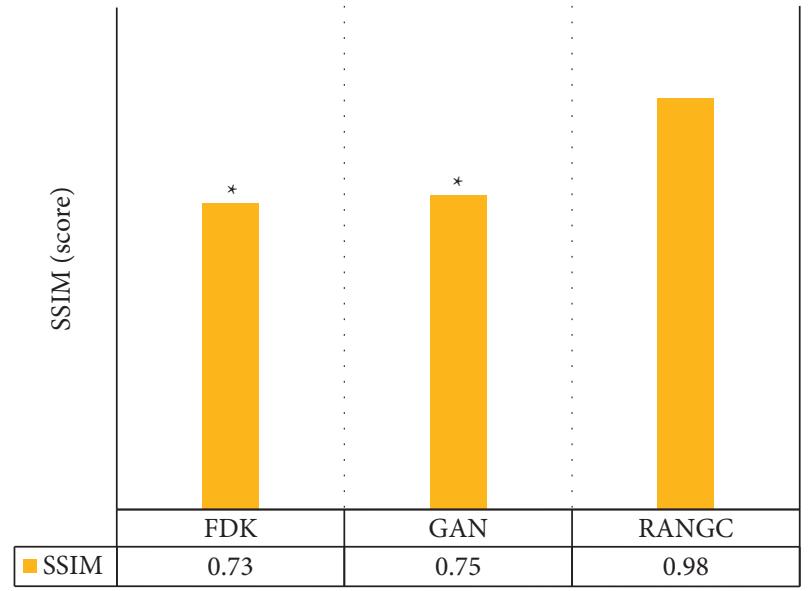

(b)

FIGURE 3: Performance analysis of the three algorithms in the scanning range $\left[0^{\circ}, 119^{\circ}\right]$. Notes: (a) was the value of PSNR and (b) was that of SSIM. ${ }^{*}$ indicated that the compared difference to RANGC was statistically significant $(P<0.05)$.

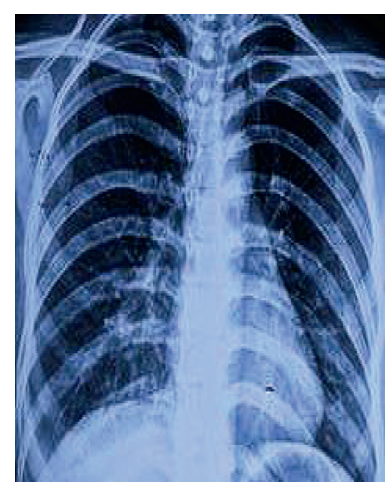

(a)

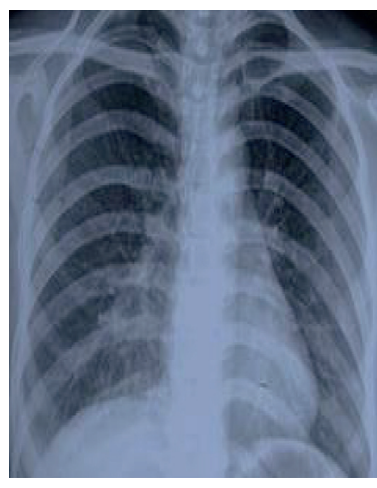

(b)

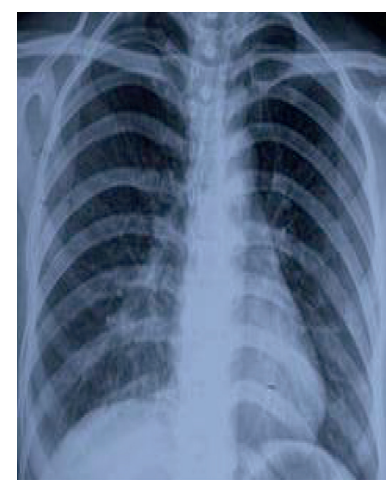

(c)

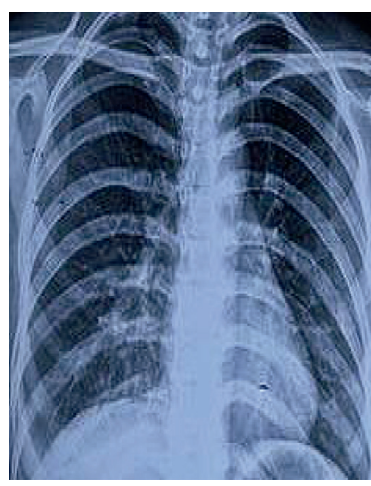

(d)

FIGURE 4: Reconstruction results of the three algorithms ((a) original image, (b) under the FDK, (c) under GAN, and (d) under RANGC). 
TABLE 1: General clinical data statistics patients in the two groups.

\begin{tabular}{lcc}
\hline Items & Test group & Control group \\
\hline Female $(n)$ & 6 & 5 \\
Male $(n)$ & 6 & 7 \\
Age (years old) & $42.65 \pm 3.6$ & $43.77 \pm 44.2$ \\
Education year (year) & $6.3 \pm 1.4$ & $6.2 \pm 1.2$ \\
\hline
\end{tabular}

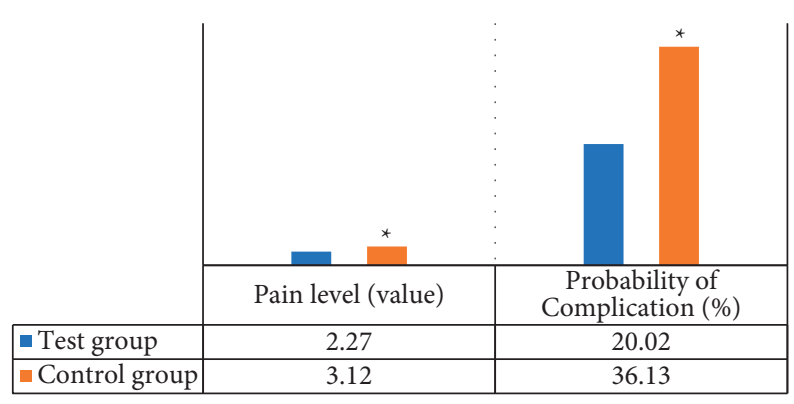

FIgURE 5: Comparation of the pain level and complication incidence of the patients in two groups. Notes: ${ }^{*}$ indicated that the comparative results were statistically significant $((P)<0.05)$.

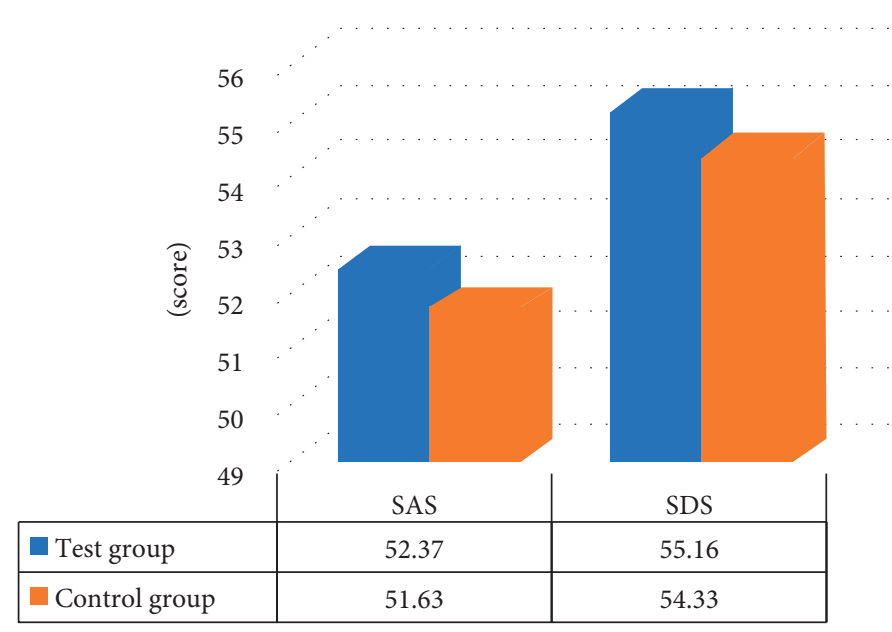

(a)

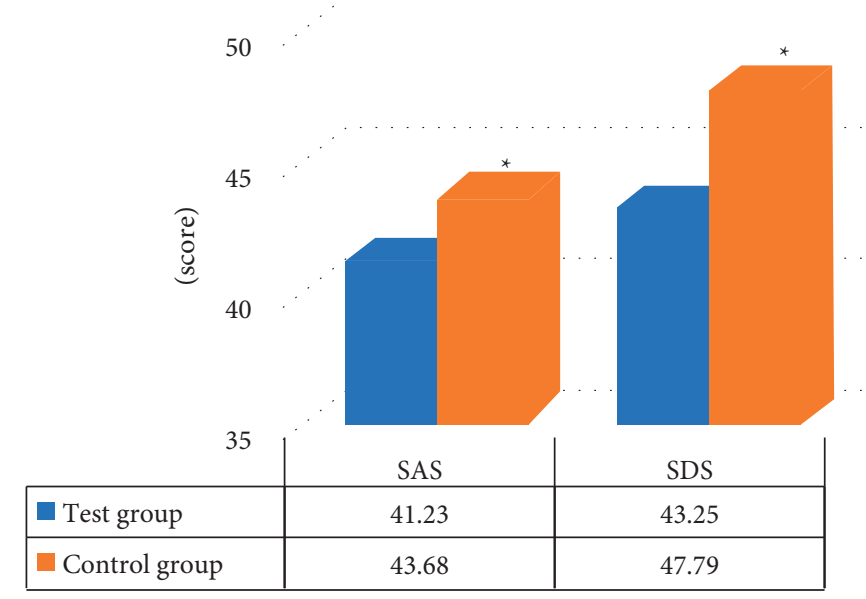

(b)

FIgure 6: Comparison of SAS and SDS before and after surgery. Notes: (a) those before the surgery; (b) those after the surgery. ${ }^{*}$ indicated that the results compared with the test group had a statistical significance $(P<0.05)$. 


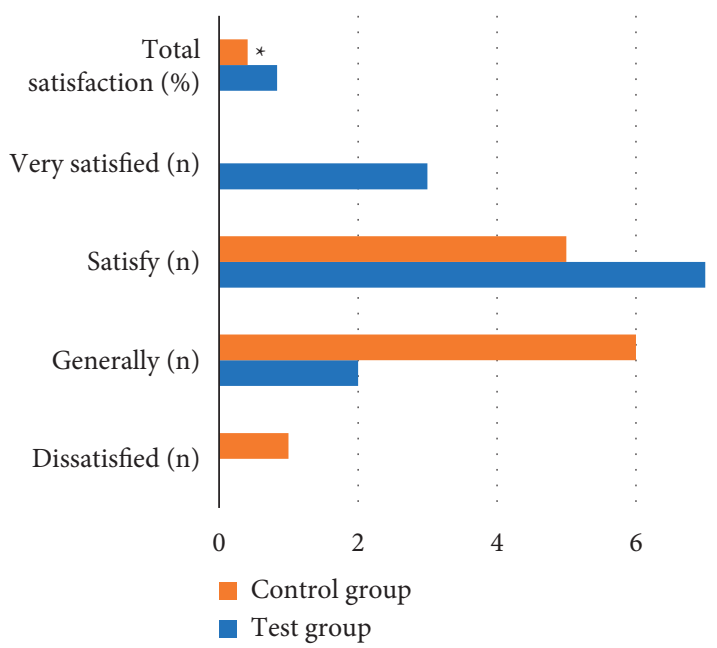

(a)

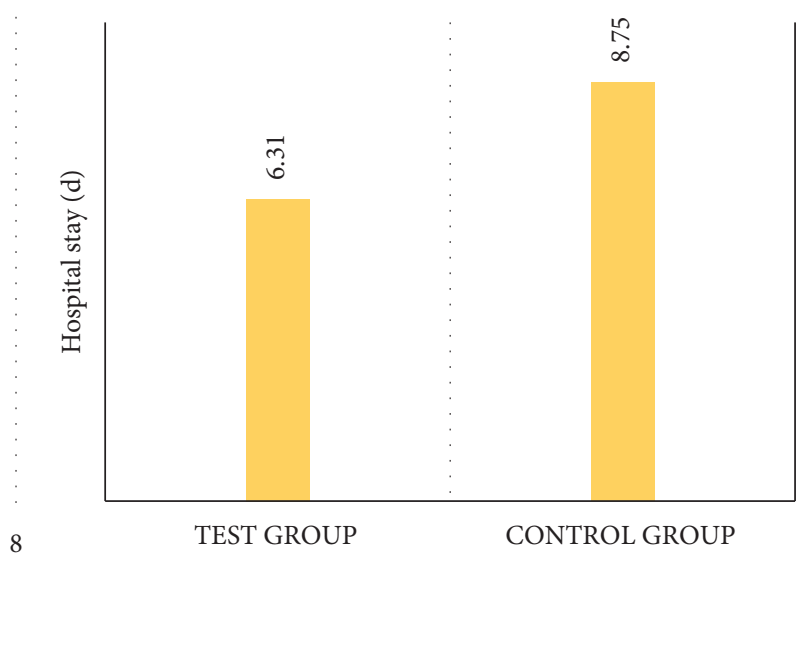

(b)

FIGURE 7: Comparison of the patients' satisfaction and duration of hospital stay in the two groups. Notes: (a) satisfaction and (b) average duration of hospital stay. ${ }^{*}$ indicated that the comparison with the test group was of statistical significance $((P)<0.05)$.

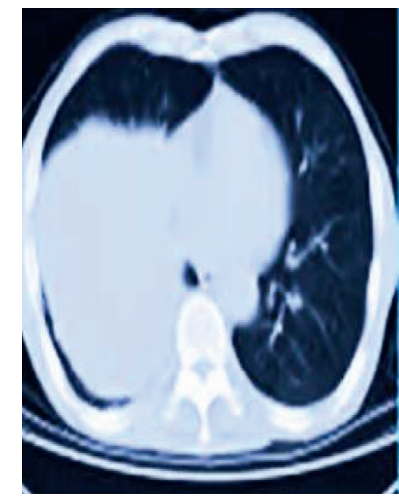

(a)

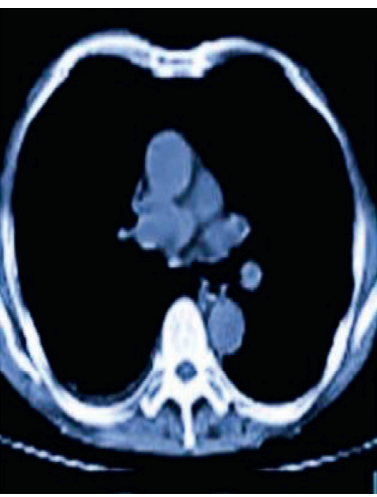

(b)

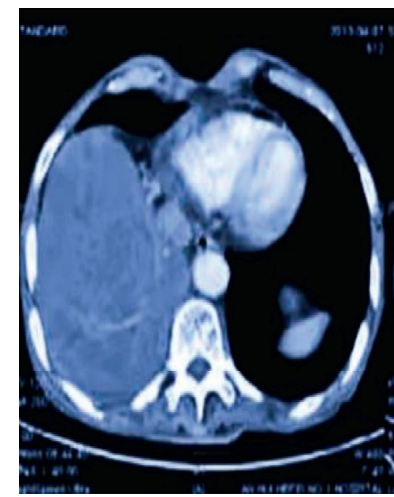

(c)

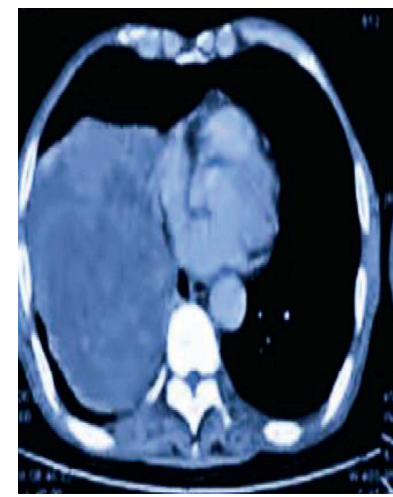

(d)

FIGURE 8: CT imaging data of the patient (the lung window shown in (a); the mediastinal window in (b); the arterial phase in (c); the venous phase in $(d))$.

3.4. CT Imaging Data of the Patients. Figure 8 shows the CT images of a 32-year-old male patient. It could be observed that there was a giant mass in the right thoracic cavity with uneven density. The medial border and posterior-lateral margin of the mass were closely attached to the mediastinum and posterior chest wall, respectively. The compressed lung edges were at the anterior edge and lateral border. The uneven and mild potentialization could be watched in the arterial and venous phases during the enhanced scanning; meanwhile, the mass envelope was incomplete.

\section{Discussion}

Giant thoracic tumors are quite rare in clinical practice, and the surgery is more complicated, difficult, and risky with many postoperative complications. Therefore, it requires careful and accurate preoperative imaging examination and CT three-dimensional reconstruction to assist the doctor in the operation $[22,23]$. The accurate three-dimensional reconstruction model can give a guide to the design of surgical incisions and the resection plan, effectively avoid surgical risks, and reduce the blindness of intraoperative operations. Thus, the purpose of reducing intraoperative bleeding and shortening the operation time can be realized [24, 25]. Therefore, the RANGC algorithm was proposed in this study and was applied to CT imaging for the impact evaluation of comfort care on the prognosis of patients with giant thoracic tumors. For the performance verification of the algorithm, the PSNR and SSIM were compared with those under the FDK and GAN algorithms, when the scanning range was $\left[0^{\circ}, 89^{\circ}\right]$ and $\left[0^{\circ}, 119^{\circ}\right]$, respectively. This was similar to the research of Kitai et al. (2020) [26]. The results showed that when the scanning range was $\left[0^{\circ}, 89^{\circ}\right]$, the PSNR and SSIM under the RANGC algorithm have the values of $45.6 \mathrm{~dB}$ and 0.99 , respectively. When the scanning range was $\left[0^{\circ}, 119^{\circ}\right]$, the PSNR and SSIM values were $39.21 \mathrm{~dB}$ and 0.98 , 
respectively. It is significantly higher than those under the FDK and GAN algorithms, and the difference was obviously of statistical significance $(P<0.05)$. Hence, the imaging quality of the RANGC algorithm was relatively the best.

For the impact of comfort care on the prognosis of giant thoracic tumor patients, the chosen patients were randomly divided into the test group and the control group. Patients in the test group received comfort care, and patients in the control group received conventional care. The gender, age, and education year of the patients were counted, and the results showed no statistically significant difference $(P>0.05)$. The pain level and complication incidence between the patients in the two groups were also compared. The average postoperative pain level of the patients in the control group was scored as 3.12 points, and the postoperative complication incidence was $36.13 \%$. For the patients in the test group, the average postoperative pain level and the postoperative complication incidence were 2.27 points and 2 $0.02 \%$, which were significantly lower than those of the control group, with the difference being statistically significant $(P<0.05)$. It was similar to the results of Yabrodi and Mastropietro (2017) [27]. Ye and Ge (2021) [28] applied comfort care to the nursing of elderly patients with advanced lung cancer, and comfort care had a positive clinical effect on these patients, improving the life quality, alleviating pain, and promoting the satisfaction of the patients. Moreover, SAS and SDS were surveyed preoperatively and postoperatively. Before the surgery, the scores of the patients in the two groups were worked out without an obvious difference. After the surgery, SAS and SDS of the patients in the test group had scores of 41.23 and 43.25 , which were greatly lower than those of the control group, and the difference was of statistical significance $(P<0.05)$. Finally, the duration of hospital stay and satisfaction of the patients in the two groups were compared. The average duration of the hospital stay of the patients in the test group was 6.31 days, and that in the control group was 8.75 days. The difference between the two groups was statistically significant $(P<0.05)$. In the test group, 3 patients felt very satisfied, 7 patients felt satisfied, and 2 people felt generally satisfied. The overall satisfaction was $83.33 \%$, while that of the control group was $41.2 \%$. The result of the control group was much lower than that of the test group, with the difference being statistically significant $(P<0.05)$. These were the same as the expected results of this study. Patients had anxiety and fear in various degrees before surgery, which caused stress responses, such as increased blood pressure and changes in immunity. With comfort care, the response plan could be formulated according to their personal psychology and own diseases to achieve patients' comfort, psychologically and physically.

\section{Conclusion}

For exploring the impact evaluation of comfort care on the prognosis of giant thoracic tumor patients with artificial intelligence algorithm-based CT imaging, the RANGC reconstruction algorithm was proposed in this study. 24 cases with giant thoracic tumors who underwent surgical treatment were selected as the research objects. The pain levels and postoperative complication incidence of patients were studied with comfort care and conventional care, respectively. It was proved that comfort care could reduce the negative emotions of patients after surgery and improve their satisfaction and life quality. However, giant thoracic tumors are clinically rare, and hence, the sample size of this study was relatively small, and the samples were of no overall representativeness. It was necessary to have the survival analysis of patients in follow-up studies. However, in general, the results of this study provided reliable data support for the clinical application of CT three-dimensional reconstruction and comfort care in the treatment of giant thoracic tumors.

\section{Data Availability}

The data used to support the findings of this study are available from the corresponding author upon request.

\section{Conflicts of Interest}

The authors declare no conflicts of interest.

\section{Acknowledgments}

This study received the funding of Qiqihar science and technology plan joint guidance project (LHYD-2021073).

\section{References}

[1] D. Yang, X. Zeng, Y. Lv, W. Liao, S. Tang, and J Jiang, "Gardner syndrome with a giant mass in the thoracic cavity: a case report and analysis of the related complications," International Journal of Clinical and Experimental Pathology, vol. 13, no. 8, pp. 2158-2162, 2020.

[2] M. Lu, Z. Zhou, Z. Lei, H. Li, and S. Boriani, "Huge myxoid chondrosarcoma expanded into the thoracic cavity with spinal involvement," European Spine Journal: Official Publication of the European Spine Society, the European Spinal Deformity Society, and the European Section of the Cervical Spine Research Society, vol. 28, no. 2, pp. 25-30, 2019.

[3] K. J. Kiser, S. Ahmed, S. Stieb et al., "PleThora: pleural effusion and thoracic cavity segmentations in diseased lungs for benchmarking chest CT processing pipelines," Medical Physics, vol. 47, no. 11, pp. 5941-5952, 2020.

[4] S. Ley and J Ley-Zaporozhan, "Bildgebung bei Mediastinaltumoren [Imaging of Mediastinal Tumors]," Zentralbl Chir, vol. 146, no. 1, pp. 88-104, 2021.

[5] S. Demura, N. Kawahara, H. Murakami et al., "Giant cell tumor expanded into the thoracic cavity with spinal involvement," Orthopedics, vol. 35, no. 3, pp. e453-6, 2012.

[6] X. Gao and J Cai, "Optimization analysis of urban function regional planning based on big data and GIS Technology," Boletin Tecnico/Technical Bulletin, vol. 55, no. 11, pp. 344-351, 2017.

[7] Y. Yang, W. Xie, Y. Ren, H. Tian, and T. Chen, “A case report of primary adrenal lymphoma," Medicine, vol. 99, no. 28, Article ID e20938, 2020.

[8] G. S. Sunam, M. Öncel, S. Ceran, K. Ödev, and H Yildıran, "Giant benign mediastinal masses extending into the pleural cavity,” Surgery Journal, vol. 10, no. 2, pp. e46-e50, 2016. 
[9] R. Abdel Jail, M. K. Abou Chaar, O. Al-Qudah, K. Abu Zahra, M. Al-Hussaini, and A. Gharaibeh, "Heart rupture repair during huge mediastinal mass resection - case report," Journal of Cardiothoracic Surgery, vol. 15, no. 1, Article ID 164, 2020.

[10] Y. Ye and J Ge, "Clinical application of comfort nursing in elderly patients with advanced lung cancer," American Journal of Tourism Research, vol. 13, no. 8, pp. 9750-9756, 2021.

[11] Z. Fang, R. Wang, M. Wang, S. Zhong, L. Ding, and S Chen, "Effect of reconstruction algorithm on the Identification of 3D printing polymers based on hyperspectral CT technology combined with artificial neural network," Materials, vol. 13, no. 8, Article ID 1963, 2020.

[12] Q. Zhang, X. Fu, K. He et al., "A new technique for the 3D reconstruction of the incisional hernia: a pilot study," Clinical Imaging, vol. 67, pp. 9 1-94, 2020.

[13] Y. He, L. Zeng, W. Yu, and C. Gong, "Noise suppressionguided image filtering for low-SNR CT reconstruction," Medical, \& Biological Engineering \& Computing, vol. 58, no. 11, pp. 2621-2629, 2020.

[14] S. Grossi, A. D’Arienzo, F. Sacchetti et al., “One-step reconstruction with custom-made 3D-printed scapular prosthesis after partial or total scapulectomy," Surgical Technology International, vol. 36, pp. 341-346, 2020.

[15] Q. Cheng, P. Sun, C. Yang, Y. Yang, and P. X. Liu, “A morphing-Based 3D point cloud reconstruction framework for medical image processing," Computer Methods and Programs in Biomedicine, vol. 193, Article ID 105495, 2020.

[16] A. Aprato, M. Olivero, G. Iannizzi, A. Bistolfi, L. Sabatini, and A. Masse, "Pelvic discontinuity in acetabular revisions: does CT scan overestimate it? A comparative study of diagnostic accuracy of 3D-modeling and traditional 3D CT scan," Musculoskeletal Surgery, vol. 104, no. 2, pp. 171-177, 2020.

[17] Z. Lv, L. Qiao, Q. Wang, and F. Piccialli, "Advanced machinelearning methods for brain-computer interfacing," IEEE/ ACM Transactions on Computational Biology and Bioinformatics, vol. 18, no. 5, pp. 1688-1698, 2021.

[18] D. Matenine, M. Schmittbuhl, S. Bedwani, P. Després, and J. A. de Guise, "Iterative reconstruction for image enhancement and dose reduction in diagnostic cone beam CT imaging," Journal of X-Ray Science and Technology, vol. 27, no. 5, pp. 805-819, 2019.

[19] C. Zhao, M. Herbst, S. Vogt et al., "Cone-beam imaging with tilted rotation axis: method and performance evaluation," Medical Physics, vol. 47, no. 8, pp. 3305-3320, 2020.

[20] L. A. Otter, K. Chen, G. Janssens et al., "Technical Note: 4D cone-beam CT reconstruction from sparse-view CBCT data for daily motion assessment in pencil beam scanned proton therapy (PBS-PT)," Medical Physics, vol. 47, no. 12, pp. 6381-6387, 2020.

[21] Z. Li, A. Cai, L. Wang et al., "Promising generative adversarial network based sinogram inpainting method for ultra-limitedangle computed tomography imaging," Sensors, vol. 19, no. 18, Article ID 3941, 2019.

[22] D. Chen, P. Wawrzynski, and Z Lv, "Cyber security in smart cities: a review of deep learning-based applications and case studies," Sustainable Cities and Society, vol. 66, Article ID 102655, 2020.

[23] Z. Wan, Y. Dong, Z. Yu, H. Lv, and Z. Lv, "Semi-supervised support vector machine for digital twins based brain image fusion," Frontiers in Neuroscience, vol. 15, no. 15, Article ID 705323, 2021.

[24] K. Pang, K. Lou, Y. Huang et al., “Application of ultrasoundguided seminal vesicle radiography combining CT three-dimensional reconstruction technique in transurethral seminal vesiculoscopy," Andrologia, vol. 52, no. 11, Article ID e13845, 2020.

[25] H. Yabuuchi, T. Kamitani, K. Sagiyama et al., "Clinical application of radiation dose reduction for head and neck CT," European Journal of Radiology, vol. 107, pp. 209-215, 2018.

[26] T. Kitai, Y. Hyodo, and H. Morikawa, "Development of CT p using deep learning based reconstruction," Japanese Journal of Radiological Technology, vol. 76, no. 1, pp. 16-25, 2020.

[27] M. Yabrodi and C. W Mastropietro, "Hypoplastic left heart syndrome: from comfort care to long-term survival," Pediatric Research, vol. 81, no. 1-2, pp. 142-149, 2017.

[28] Y. Ye and J. Ge, "Clinical application of comfort nursing in elderly patients with advanced lung cancer," American journal of translational research, vol. 13, no. 8, pp. 9750-9756, 2021. 